the 9 patients with renal remission, 4 relapsed. Finally, 4 patients required dialysis, including 1 death.

Conclusion The presence of intrarenal B cells rarely forms TLO in lupus nephritis. Due to the small sample size, we were unable to determine their prognostic role. Nevertheless, we report here the longer time to renal remission for grade 3 and 4 infiltrates; prospective studies with repeated renal biopsies are needed to better characterize their relationship to disease progression.

Funding None

\section{O22 CORRELATION BETWEEN INTERSTITIAL CD8+ T CELL INFILTRATION AND FIBROTIC PROCESSES IN A MOUSE MODEL OF LUPUS NEPHRITIS}

1,2 Pauline Montigny, ${ }^{1}$ Aurélie Degroof, ${ }^{3}$ Davide Brusa, ${ }^{1,4}$ Frédéric Houssiau,
${ }^{1,4}$ Bernard Lauwerys. ${ }^{1}$ Pôle de Pathologies Rhumatismales Systémiques et Inflammatoires,
UCLouvain, Louvain; ${ }^{2}$ Service de Rhumatologie, CHU UCL Namur, Yvoir; ${ }^{3}$ Plateforme de
Cytométrie de Flux, Institut de Recherche Expérimentale et Clinique, UCLouvain, Louvain;
${ }^{4}$ Service de Rhumatologie, Cliniques Universitaires Saint Luc, Bruxelles, Belgium

10.1136/lupus-2020-eurolupus.33

Background Tubulo-interstitial damage during lupus nephritis $(\mathrm{LN})$ is associated with poor renal prognosis in the long run. Here, we describe the progression of tubulo-interstitial fibrosis and immune cells infiltration with emphasis on CD8 + T cells, in parallel with renal outcomes in a mouse lupus model.

Methods We collected blood, urine and kidneys from 39 B6/ Sle1.Sle2.Sle3 lupus-prone mice, before disease onset and at different stages of disease progression. RNA was extracted from kidneys and hybridized on Mouse Gene 2.0 ST exon arrays. Histopathological scores (NIH Activity and Chronicity Indexes) and digital quantification of fibrosis, IgGK deposits, $\mathrm{CD} 8+$ and $\mathrm{CD} 3+\mathrm{T}$ cells were performed on total kidney. Renal CD8 $+\mathrm{T}$ cell phenotypes were determined by flow cytometry. Plasma urea and albuminuria were measured by immunoenzymatic assays.

Results IgGк deposits, CD8 $+\mathrm{T}$ cell infiltration and interstitial fibrosis increase with the progression of renal disease, evaluated by histopathological scores and plasma urea. Further, digital quantifications allowed us to identify a significant correlation $(\mathrm{r}=0,45, p=0,011)$ between local CD8 $+\mathrm{T}$ cell population and fibrosis, while total $\mathrm{CD} 3+$ cells population and

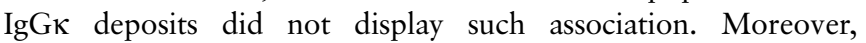
characterization of $\mathrm{CD} 8+\mathrm{T}$ cell subpopulations showed that fibrosis is more specifically linked to effector functions of
CD8 $+\mathrm{T}$ cells. Transcriptomic analyses supported this association, with a high correlation coefficient between mean expression of effector functions transcripts and the presence of a fibrotic signature $(r=0,92, p<0,0001)$.

Conclusions Our results support the association between CD8 $+\mathrm{T}$ cell tubulo-interstitial infiltration and renal outcomes in a mouse lupus model. Further, a strong correlation is identified between effector functions of CD8 $+\mathrm{T}$ cells and fibrotic processes, opening new avenues of research in the pathogenesis of LN.

Acknowledgements This work was supported by F.R.S-F.N.R.S and by Fondation Roi Baudouin

\section{O23 IDENTIFICATION OF PROTEIN-QUANTITATIVE TRAIT LOCI (PQTLS) IN THE INTERFERON SIGNALLING PATHWAY}

Christian Lundtoft, Pascal Pucholt, Johanna K Sandling, Lars Rönnblom, Niklas Hagberg. Dept. of Medical Sciences, Rheumatology, Uppsala University, Uppsala, Sweden

\subsection{6/lupus-2020-eurolupus.34}

Background Interferon (IFN)- $\alpha$ and IFN- $\gamma$ are important cytokines in the pathogenesis of systemic lupus erythematosus (SLE), and several of the genetic associations with SLE are found in genes that are fundamental for the IFN response (e. g. TYK2, STAT4, IRF5). This study aimed to define the genetic regulation of the IFN system, and to link disease-associated SNPs to alterations in the IFN system.

Methods Peripheral blood mononuclear cells from 303 healthy individuals were stimulated with IFN- $\alpha$ or IFN- $\gamma$ (figure 1). Basal levels of IFN-receptors (IFNAR2 and IFNGR1) and IFN-induced phosphorylation of STAT1 and STAT4, expression of CXCL9, CXCL10, HLA-ABC and HLA-DRPQ was determined in 6 cell subsets using flow cytometry. Each readout was mapped as a pQTL using 3.4 million SNPs with a minor allele frequency $\geq 5 \%$ (Illumina Global Screening Array with subsequent genome-wide imputation) in an additive model correcting for covariates. pQTLs were probed for overlap with GWAS SLE-associated SNPs.

Results We identified 8 genome-wide significant pQTLs $(\mathrm{p}<5.0 \mathrm{e}-8), 3$ of which were associated with basal IFN receptor levels, 3 with IFN- $\alpha$, and 2 with IFN- $\gamma$-induced traits. One pQTL affected protein expression in cis (IFNAR2), whereas the other were trans-pQTLs. The strongest association was observed for a SNP in HLA-A that affected IFNAR2 level

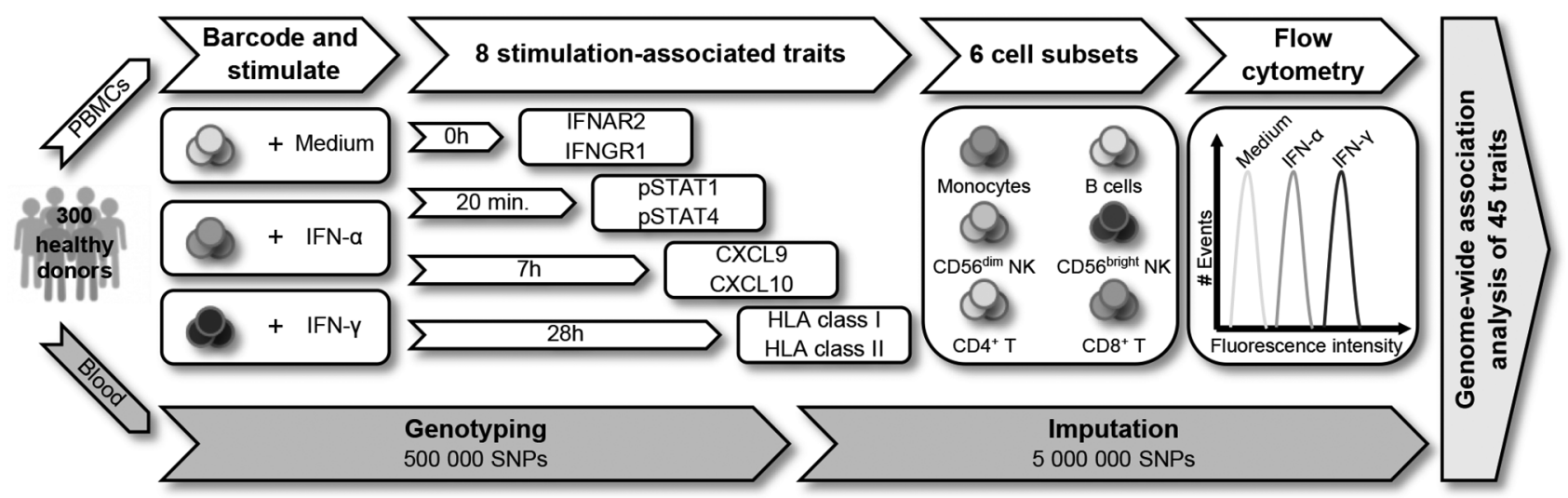

Abstract 023 Figure 1 Graphical study design 
in $B$ cells $(p=3 e-26), C D 8^{+} T$ cells $(3 e-12)$ and $C D 4^{+} T$ cells $(\mathrm{p}=9 \mathrm{e}-10)$.

A trans-pQTL for IFNGR1 level in monocytes (rs1801274 in FCGR2A; $\mathrm{p}=3 \mathrm{e}-23$ ) and a suggestive significant $\mathrm{pQTL}$ $(\mathrm{p}<5.0 \mathrm{e}-5)$ for IFN- $\gamma$-induced STAT1 phosphorylation in monocytes (rs912784 in LRRC63) were previously associated with SLE. Notably, none of the SNPs in TYK2, STAT4 or IRF5 reached the suggestive significant levels for the parameters studied, and no enrichment of SLE-associated SNPs were identified among pQTLs.

Conclusions We demonstrate a cell-type and stimuli-specific genetic regulation of the IFN system. Two SNPs previously linked to SLE were associated with alterations in the IFN- $\gamma$ receptor expression or response. Further studies to determine the underlying mechanisms of these associations are ongoing.

\section{O24 FLARE ASSESSMENTS IN PATIENTS WITH ACTIVE SYSTEMIC LUPUS ERYTHEMATOSUS (SLE) TREATED WITH ANIFROLUMAB IN 2 PHASE 3 TRIALS}

${ }^{1}$ Richard Furie, ${ }^{2}$ Eric $\mathrm{F}$ Morand, ${ }^{3}$ Anca Askanase, ${ }^{4,5} \mathrm{Ed}$ Vital, ${ }^{6}$ Rubana N Kalyani, ${ }^{7}$ Gabriel Abreu, ${ }^{6}$ Lilia Pineda, ${ }^{6}$ Raj Tummala. 'Zucker School of Medicine at Hofstral Northwell, Great Neck, NY, USA; ${ }^{2}$ Monash University, Melbourne, VIC, Australia; ${ }^{3}$ Columbia University College of Physicians and Surgeons, New York, NY, USA; ${ }^{4}$ University of Leeds, Leeds, UK; ${ }^{5}$ NIHR Leeds Biomedical Research Centre at Leeds Teaching Hospitals NHS Trust, Leeds, UK; ${ }^{6}$ AstraZeneca, Gaithersburg, MD, USA; ${ }^{7}$ AstraZeneca, Gothenburg, Sweden

10.1136/lupus-2020-eurolupus.35

Background Anifrolumab treatment improved BICLA response rates in patients with SLE in the phase 3 TULIP-2 and
TULIP-1 trials (Morand et al, 2020; Furie et al, 2019). In addition, annualized flare rates were lower with anifrolumab vs placebo. TULIP-2 and TULIP-1 data were analyzed to assess effects of anifrolumab on the number of flares and time to first flare during 52 weeks of treatment.

Methods The randomized, double-blind, placebo-controlled TULIP-2 and TULIP-1 trials evaluated efficacy and safety of intravenous anifrolumab (300 mg Q4W) over 52 weeks in patients with moderate to severe SLE despite standardof-care treatment. Flares were defined as $\geq 1$ new BILAG2004 A or $\geq 2$ new (worsening) BILAG-2004 B domain scores compared with the prior month's visit. Number of flares, time to first flare, and annualized flare rate were assessed.

Results In TULIP-2 (anifrolumab, $\mathrm{n}=180$; placebo, $\mathrm{n}=182$ ) and TULIP-1 (anifrolumab, $\mathrm{n}=180$; placebo, $\mathrm{n}=184$ ), fewer anifrolumab-treated patients experienced $\geq 1$ flare (TULIP-2: 31.1\%, $\mathrm{n}=56$; TULIP-1: $36.1 \%, \mathrm{n}=65)$ vs placebo-treated patients (TULIP-2: $42.3 \%, \mathrm{n}=77$; TULIP-1: 43.5\%, $\mathrm{n}=80$ ). Results favoring anifrolumab were observed in time to first flare (TULIP-2: hazard ratio $[\mathrm{HR}]=0.65,95 \%$ confidence interval [CI] 0.46-0.91; TULIP-1: HR $=0.76$, 95\% CI $0.55-$ 1.06; figure 1) and annualized flare rates (TULIP-2: adjusted rate ratio $=0.67,95 \%$ CI $0.48-0.94 ;$ TULIP-1: rate ratio $=0.83,95 \%$ CI $0.60-1.14)$.

Conclusions Across 2 phase 3 trials, we observed reduced total number of flares and annualized flare rates, as well as prolongation of time to first flare with anifrolumab treatment vs placebo. These results support the potential of anifrolumab to reduce disease activity and reduce flares, benefiting patients with SLE.

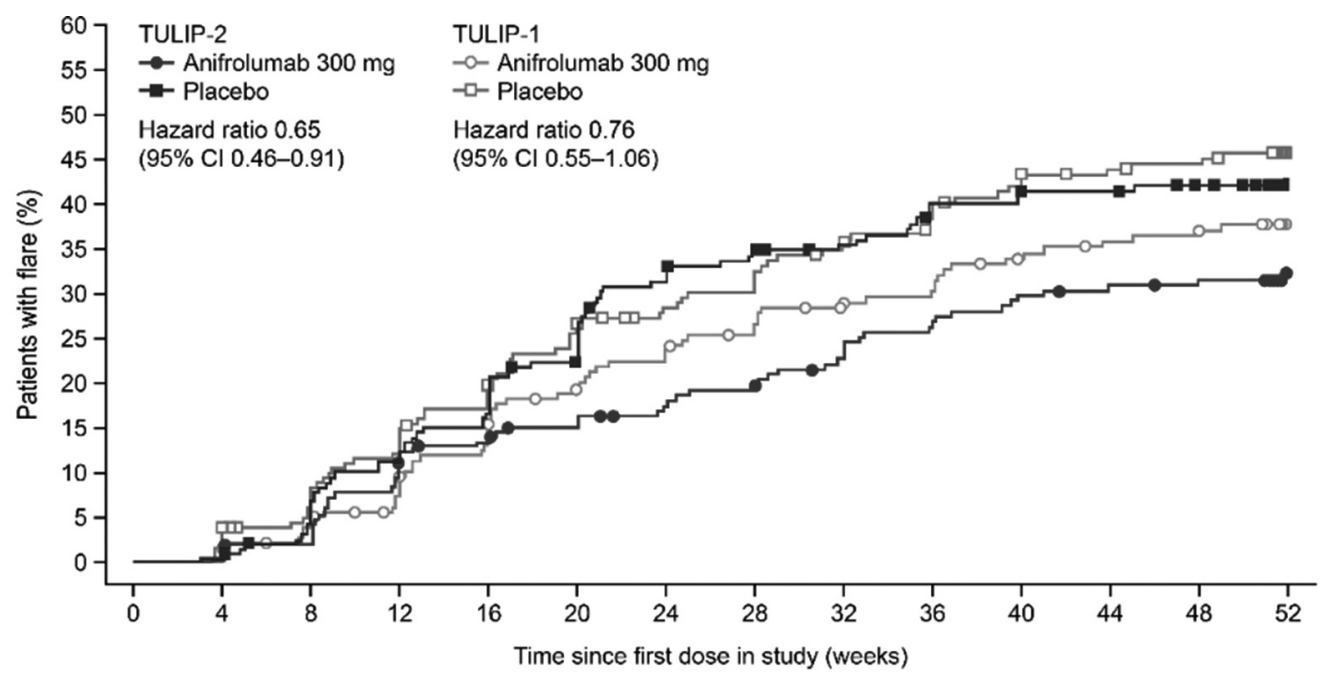

\begin{tabular}{|rllllllllllllll|}
\hline \begin{tabular}{|} 
Number at risk in TULIP-2 \\
Anifrolumab 300 mg
\end{tabular} 180 & 179 & 175 & 158 & 153 & 147 & 141 & 138 & 130 & 124 & 118 & 116 & 114 & 103 \\
Placebo & 182 & 181 & 167 & 159 & 149 & 137 & 120 & 113 & 107 & 98 & 97 & 95 & 91 & 77 \\
\hline
\end{tabular}

BILAG, British Isles Lupus Assessment Group.

Note: Flare defined as $\geq 1$ new BILAG-2004 A or $\geq 2$ new (worsening) BILAG-2004 B domain scores as compared with the previous visit. Time to first flare is derived as date of first flare minus date of first administration of investigational product. If the patient did not have a flare, the time to flare is censored at the end of the exposure time. Time to first flare was evaluated using a Cox proportional-hazards model. 